\title{
Placental Apoptosis in Small for Gestational Age Babies: A Comparison between Swedish and Pakistani Populations
}

\author{
Shahzad Akram ${ }^{1, *}$, Eleni Simatou ${ }^{1}$, Jan-Bernd Stukenborg ${ }^{1}$, Lars Hagenäs ${ }^{1}$, \\ Zulfiqar A. Bhutta ${ }^{2}$ and Olle Söder ${ }^{1}$
}

\author{
${ }^{1}$ Department of Women's and Children's Health, Paediatric Endocrinology Unit Q2:08, Karolinska Institutet \\ and University Hospital, SE-17176 Stockholm, Sweden
}

${ }^{2}$ Department of Paediatrics, The Aga Khan University, P.O. Box 3500, Karachi 74800, Pakistan

\begin{abstract}
Background: Foeto-placental growth is regulated by a complex balance of growth promoting and growth inhibiting factors and hormones, namely the insulin-like growth factors (IGF) and the intracellular caspase proteins. Changes in the IGF-axis appear to affect this balance, with deficiencies possibly triggering apoptosis.

Aim: To ascertain levels of apoptosis in the placenta of infants born small for gestational age (SGA) and appropriate for gestational age (AGA), comparing samples from two population groups, Pakistani and Swedish, in an attempt to better understand the mechanism behind foetal-placental growth restriction.

Methods: Placental samples were taken immediately following delivery in both Karachi and Stockholm. In total 36 samples were included for further analysis (Pakistani: SGA $n=12$, AGA $n=12$; Swedish: SGA $n=7$, AGA $n=5$ ). Protein extraction was conducted for cell-death ELISA, and the remaining tissue samples were then paraffin embedded for further immunohistochemical and immunoflourescent analysis, looking at the apoptotic proteins, p53, caspase 8, and caspase 3. Furthermore, we compared maternal and newborn anthropometry between populations.

Results: A higher apoptotic index, for caspase 8 and caspase 3, was seen in the Pakistani samples, as compared to the Swedish samples $(p<0.01)$. TUNEL assays showed higher levels of apoptosis in the Pakistani population as compared to the Swedish population $(p<0.01)$. Cell death ELISA analysis showed greater apoptotic activity in placenta from the Pakistani population as compared to the Swedish groups $(p<0.05)$ as well as increased apoptotic activity in the SGA groups as compared to the AGA groups within each population (ELISA, $p<0.05$ ). No differences were seen in p53 levels as assessed by immunohistochemistry. Pakistani mothers were, on average, shorter than their Swedish counterparts $(p<0.01)$.

Conclusion: Increased apoptotic activity in placenta of the Pakistani population, as compared to their Swedish counterparts, may be associated with decreased foetal-placental growth seen in this population, particularly in babies born SGA. These findings, along with previously published results of the IGF-axis, and birth weight outcomes, suggests that lower IGF levels may be involved in the extracellular triggering of apoptosis, through caspase 8 . This may further suggest a possible mechanism of foetal-placental growth restriction.
\end{abstract}

Keywords: SGA, AGA, Apoptosis, Caspase 3, Caspase 8.

\section{INTRODUCTION}

Foetal growth restriction (FGR) is a complex problem of pregnancy with many short and long term consequences. These include peri-natal morbidity and mortality, and long-term health complications, including cardiovascular disease. There are multiple aetiologies underlying this vast clinical problem, though the exact molecular mechanisms are still unknown. FGR further leads to babies being born small for their gestational ages (SGA) affecting an estimated 30 million pregnancies each year [1]. Placental, and foetal, growth regulation is a complex process controlled by genetic, environmental and hormonal factors [2]. The hormonal factors include growth hormone axes and their receptors. A key regulatory axis of normal growth

*Address correspondence to this author at the Paediatric Endocrinology Unit Q2:08, Department of Women's and Children's Health, Astrid Lindgren's Children's Hospital, Karolinska Institutet and University Hospital, SE - 17176 Stockholm, Sweden; Tel: (+) 46851772 372; Fax: (+) 46851775 128; E-mail: Shahzad.Akram@ki.se is the insulin-like growth factor (IGF) hormone axis, comprised of the insulin-like growth factors (IGF-I and IGF-II), the IGF binding proteins (IGFBPS 1-6), IGFBP proteases, and the IGF-I receptor. Deficiencies, or excesses, of this axis thus affect foetal growth outcomes and viability [3].

Cellular and tissue growth is further regulated by a balance of growth promoting and suppressing factors. Changes in this balance may therefore tip growth in either direction, as can be seen with decreases of IGF-I and IGF-II leading to SGA in pregnancy [4]. Furthermore, increases in placental apoptosis have also been seen in pregnancies affected with the clinical syndrome pre-eclampsia, which is widely associated with growth inhibition [5]. Though the exact mechanism is unknown, placental apoptosis is believed to be a key regulatory element in foetal-placental growth [6]. The two major pathways of apoptosis are the intrinsic and extrinsic pathways. The intrinsic pathway is regulated through intra-cellular mitochondrial stress with 
subsequent activation of the B-cell lymphoma 2 and apoptotic protease activating factor 1 (BCL-2/APAF1) pathway, leading to the nuclear activation of the caspase family of enzymes with subsequent cellular destruction. The extrinsic pathway is stimulated through cell surface tyrosine-kinase receptors with direct downstream activation of the caspase enzymes. Activation of either of these pathways triggers apoptosis leading to controlled cell and tissue death [7].

A principal regulator of apoptosis is protein 53 ( $\mathrm{p} 53$, pro-apoptotic), which plays a role in tumour genesis. Down regulation of the gene results in decreased apoptosis and increased cell and tissue growth [8]. Levels of p53 and the activator protein, $\mathrm{Bcl}-2$, can therefore be used as markers to assess the intrinsic activation of the apoptotic cascade. In contrast, the extrinsic pathway is mediated through cell surface death receptors, leading to activation of caspase 8 [9]. Levels of caspase 8 may therefore be used as markers of the extrinsic activation of the apoptotic cascade. Aside from the regulators of apoptosis, certain key executor proteins may be seen in increased programmed cell death. Caspase 3 is one particular down-stream executioner protein of importance, as it is activated by both the intrinsic and extrinsic pathways and can be used to differentiate apoptosis from necrosis [10].

The aim of this study was to analyse components of the apoptotic axes, namely p53, caspase 8, and caspase 3 , in the placenta of babies born small and appropriate for their gestational ages (SGA and AGA, respectively), in the Pakistani and Swedish populations in an attempt to further understand the mechanisms behind foetal growth restriction.

\section{METHODS}

\section{Population Demographics}

Following informed and written consent, thirty-six placental samples were taken from two populations immediately after delivery. The samples were placed in RNAlater ${ }^{\circledR}$ to stabilise RNA, DNA, and proteins, and subsequently frozen for further storage and transport. Categorization of samples for further analysis was done based on the respective newborn birth weights. Inclusion criteria in the AGA groups included: term delivery, normal birth weights, exclusion of any clinical diseases/syndromes, and normal progression of pregnancy. Inclusion criteria in the SGA groups included all pregnancies that had a birth weight of less than the $10^{\text {th }}$ percentile for their gestational ages $\left(\leq 10^{\text {th }}\right.$ percentile, based on population specific growth charts). Twelve Swedish samples (7 growth restricted, and 5 normal controls/appropriate for gestational age, AGA), and 24 Pakistani samples (12 growth restricted, and 12 normal controls/AGA), were included for further analysis, as described below. Population demographics are as previously described [11, 12]. Infant and maternal height, weight, and body mass index (BMI) at delivery were measured.

\section{Cell Death ELISA}

Protein extraction and quantification was conducted according to the Bradford assay (Bradford protein assay kit, Bio-Rad Laboratories, CA, USA). Cellular apoptosis was assessed by the detection and quantification of cytoplasmic histone-associated DNA fragments (mono- and oligonucleosomes) by photometric enzyme immunoassay (Cell Death Detection Kit ELISA ${ }^{\text {PLUS }}$, Roche Diagnostics Scandinavia $A B$ ). Tissue lysis was performed according to the manufacturer's protocol $(20 \mu \mathrm{l}$ sample $+80 \mu \mathrm{l}$ lysis buffer). Following centrifugation, the supernatant was then removed for analysis of cytoplasmic histone-associated DNA fragments. Necrosis was excluded by the negative determination of mono- and oligonucleosomes in the protein extract from tissue samples. All samples were analysed in triplicates. Results are expressed as 'apoptotic activity', an optical absorbance ratio of the absorbance $\left(10^{-3}\right)$ of the dead/dying cells divided by the absorbance from the corresponding positive and negative controls (enrichment factor ratio, based on manufacturer's protocol).

\section{Immunohistochemical Staining}

Prior to immunohistochemical analysis, samples were defrosted and fixed in $4 \%$ paraformaldehyde for 24 hours following which they were transferred to $70 \%$ ethanol at $4^{\circ} \mathrm{C}$. The samples were then trimmed and routinely embedded in paraffin using an automated processor, sectioned at $5 \mu \mathrm{m}$ thickness. Three sections of each sample were included on a single slide. Immunohistochemical staining for $p 53$, caspase 8 , and caspase 3, was conducted using the BOND-MAX analyser (Leica Microsystems, Germany). Endogenous peroxidase activity was quenched for $10 \mathrm{~min}$ at room temperature in methanol containing $1.5 \%$ hydrogen peroxide. Antigen retrieval was done in the buffer ERI (citrate-based, $\mathrm{pH}$ 5.9-6.1) for 20 minutes at $94^{\circ} \mathrm{C}$ to $96^{\circ} \mathrm{C}$. The automated analyser was then programmed 
to run the primary antibody for 30 minutes. The antibody DO-7 (p53; Dako Sweden AB), was used at a dilution of 1:500, the antibody AB61755 (caspase 8; Abcam PLC, UK), was used at a dilution of 1:500, and the antibody JHM62 (caspase 3; Novocastra, UK) was used at a dilution of 1:50. The sections were washed and then incubated with goat anti-rabbit immunoglobulins conjugated to peroxidase-labelled dextran polymer (Dako Sweden $A B$ ) for 15 minutes at room temperature. The peroxidase reaction was then developed with 3'3-diaminobenzidine (DAB) and counterstained with haematoxylin, for 10 minutes each, respectively (Figures 1 and 2). Staining for each respective antibody was done as a single experiment in the BOND-MAX analyser. Positive controls were as follows: intestine (p53); testis (caspase 8); skin (caspase 3).

\section{TUNEL Assay}

To examine DNA fragmentation by apoptosis we used terminal deoxynucleotidyl transferase dUTP nick end labelling (TUNEL) by labelling the terminal end of nucleic acids. Placental samples were paraffin embedded and fixed as described above. Sections were treated with xylene for 10 minutes, then, gradient ethanol concentration from $100-70 \%$, each for 5 minutes. Afterwards, samples were washed with 1XPBS and treated with $0.1 \%$ sodium citrate + $0.1 \%$ Triton $\mathrm{X}-100$ in $1 \mathrm{xPBS}$ for 8 minutes at room temperature. In Situ Cell Death Detection Kit (Roche) was used according to the protocol provided. The samples were mounted and counterstained for $15 \mathrm{~min}$ in VECTASHIELD ${ }^{\circledR}$ with DAPI (Vector), and the resulting fluorescent signals were detected by fluorescence microscopy.

\section{Image Analysis and Scoring}

Ten separate fields in each of three nonadjacent sections from each sample (per slide) were examined for staining employing a Leica microscope $(40 \mathrm{X}$ magnification; Leica Microsystems $\mathrm{GmBH}$, Wetzlar, Germany) equipped with a Sony digital video camera
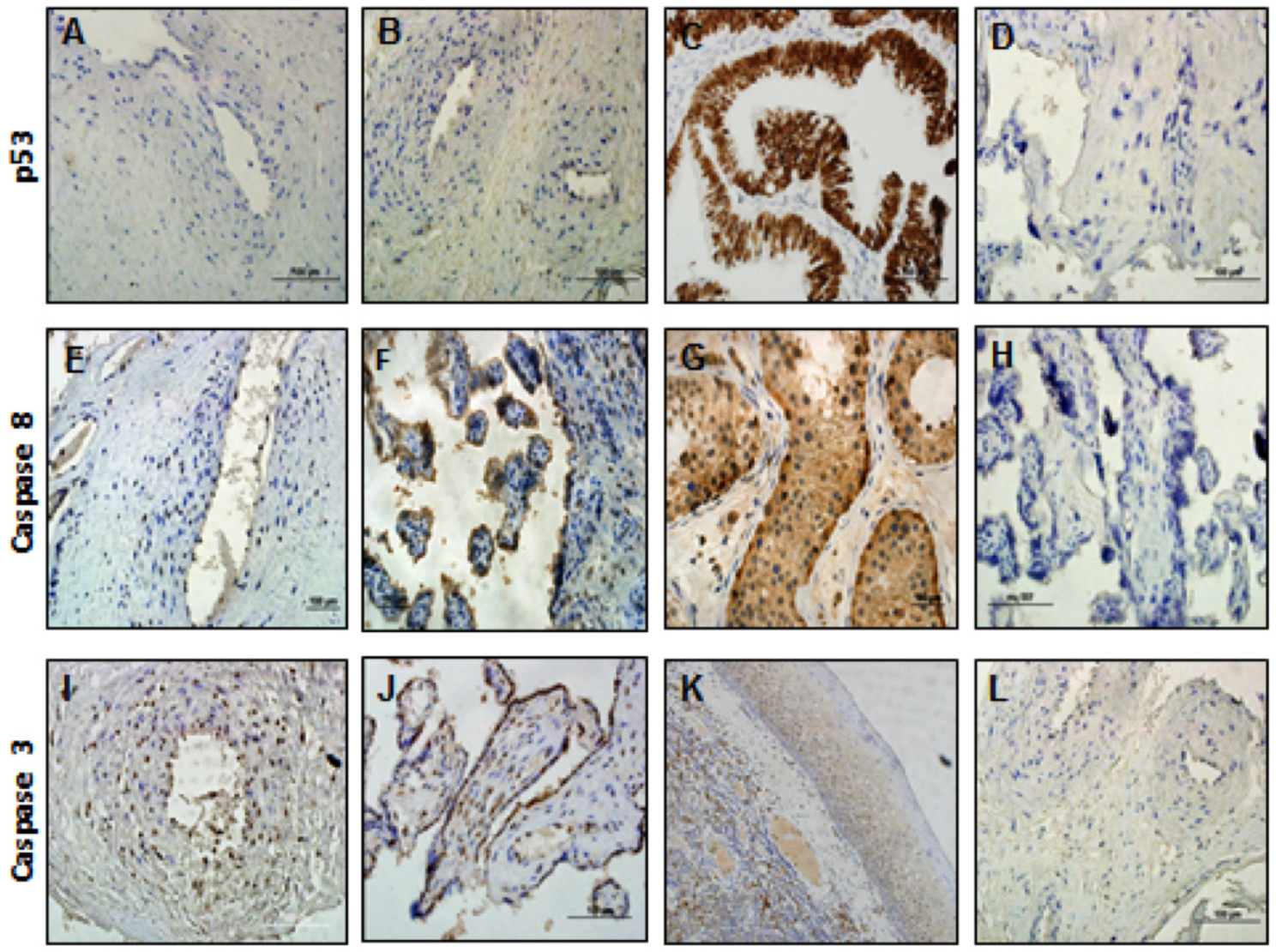

Figure 1: Immunohistochemical staining showing positive (brown) and negative (blue) cells for p53, caspase 8 and caspase 3 , comparing small and appropriate for gestational age placental samples (SGA and AGA, respectively), in the Pakistani population. Positive controls were as follows: intestine (p53); testis (caspase 8); skin (caspase 3): (A) p53 AGA group; (B) p53 SGA group; (C) p53 positive control; (D) p53 negative control; (E) Caspase 8 AGA group; (F) Caspase 8 SGA group; (G) Caspase 8 positive control; (H) Caspase 8 negative control; (I) Caspase 3 AGA group; (J) Caspase 3 SGA group; (K) Caspase 3 positive control; (L) Caspase 3 negative control. 

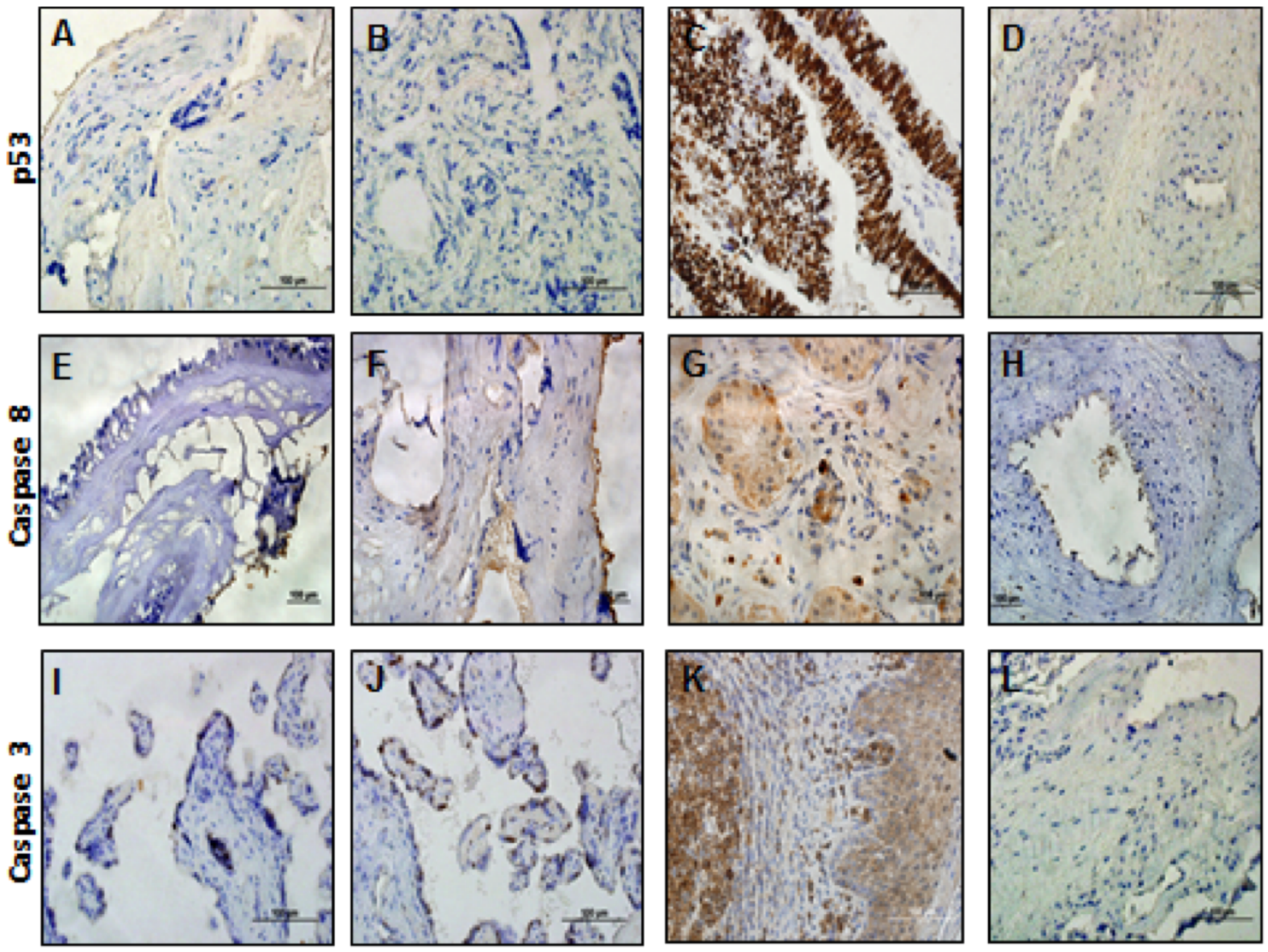

Figure 2: Showing similar immunohistochemical staining in the Swedish population. Positive controls were as follows: intestine (p53); testis (caspase 8); skin (caspase 3): (A) p53 AGA group; (B) p53 SGA group; (C) p53 positive control; (D) p53 negative control; (E) Caspase 8 AGA group; (F) Caspase 8 SGA group; (G) Caspase 8 positive control; (H) Caspase 8 negative control; (I) Caspase 3 AGA group; (J) Caspase 3 SGA group; (K) Caspase 3 positive control; (L) Caspase 3 negative control. As can be seen in the figures, p53 stained negatively for both populations. Furthermore, relatively more positive cells were seen in the SGA group as compared to the AGA group for both caspase 8 and caspase 3 . The Pakistani placental samples showed a higher apoptotic index as compared to the Swedish placental samples $(p<0.01)$.

(Diagnostic Instruments, Inc., Sony, Japan) interfaced to a computer. For the purposes of accurate image analysis, immunostained cells were manually evaluated by two blinded observers to the groups (S.A \& E.S). A quantitative manual scoring system was used counting the total number of (-) negative and (+) positive staining cells in each of the fields. Counts from both observers were then averaged and are as described as a ratio/percentage of positive cells. These results are expressed as an apoptotic index (relative ratio of the apoptotic cells to the total number of cells).

\section{Ethical Considerations}

All placental samples were obtained following ethical approval from the Northern Stockholm Ethical Committee, Stockholm, Sweden, and the Aga Khan University Ethical Review Committee, Karachi, Pakistan. Furthermore, written consent was obtained from all mothers prior to sample acquisition.

\section{Statistical Analysis}

Statistical comparisons between the groups were done using Student's t-test. Further comparisons were done using analysis of variance (ANOVA), and the Tukey's post hoc test. Statistical significance was taken as a $p$-value of less than $5 \%(p<0.05)$.

\section{RESULTS}

\section{Population Demographics}

The study group consisted of samples from both the Swedish and Pakistani populations, as previously described [11]. In total, 24 Pakistani samples and 12 Swedish samples were included in the study (Table 1). Swedish mothers were significantly taller than the Pakistani mothers in the appropriate for gestational age (AGA) group $(p<0.01$; Table 1a). Swedish mothers were also both significantly taller and weighed more 
Table 1: Population demographics showing newborn and maternal height, weight, and body mass index (BMI) at delivery. (1a) Comparison of the anthropometric variables in the appropriate for gestational age (AGA) groups in the Pakistani $(n=12)$ and Swedish $(n=5)$ populations. (1b) Comparison of anthropometric variables between small for gestational age (SGA) groups in both populations (Pakistani: $n=12$; Swedish $n=7$ ). The corresponding p-values are given (Student's t-test). A p-value of $<0.05$ is considered statistically significant. Anthropometric data for each of the populations is as previously published and is used here for comparative purposes only [12]

\begin{tabular}{|c|c|c|c|c|}
\hline \multicolumn{2}{|c|}{ 1a AGA } & 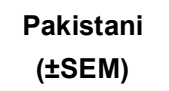 & $\begin{array}{l}\text { Swedish } \\
\text { (士SEM) }\end{array}$ & p-value \\
\hline \multirow{3}{*}{ Newborn } & Height (m) & $0.49(0.01)$ & $0.49(0.04)$ & 0.82 \\
\hline & Weight (kg) & $3.15(0.09)$ & $3.23(0.09)$ & 0.38 \\
\hline & Body Mass Index & $12.98(0.19)$ & $13.03(1.00)$ & 0.89 \\
\hline \multirow{3}{*}{ Maternal } & Height (m) & $1.51(0.01)$ & $1.65(0.03)$ & $<0.01$ \\
\hline & Weight (kg) & $55.51(1.05)$ & $70.81(3.54)$ & 0.14 \\
\hline & Body Mass Index & $23,28(0.41)$ & $25.96(1.12)$ & 0.97 \\
\hline
\end{tabular}

\begin{tabular}{|c|c|c|c|c|}
\hline \multicolumn{2}{|c|}{ 1b SGA } & $\begin{array}{c}\text { Pakistani } \\
\text { (士SEM) }\end{array}$ & $\begin{array}{l}\text { Swedish } \\
\text { ( } \pm \text { SEM) }\end{array}$ & $p$-value \\
\hline \multirow{3}{*}{ Infant (birth) } & Height (m) & $0.41(0.01)$ & $0.44(0.09)$ & 0.31 \\
\hline & Weight $(\mathrm{kg})$ & $1.78(0.09)$ & $2.00(0.17)$ & 0.06 \\
\hline & Body Mass Index & $10.39(0.46)$ & $10.03(0.68)$ & 0.74 \\
\hline \multirow{3}{*}{ Maternal } & Height (m) & $1.51(0.01)$ & $1.66(0.03)$ & $<0.01$ \\
\hline & Weight $(\mathrm{kg})$ & $47.41(0.92)$ & $60.18(2.01)$ & $<0.01$ \\
\hline & Body Mass Index & $20.80(0.39)$ & $22.27(0.56)$ & 0.06 \\
\hline
\end{tabular}

than their Pakistani counterparts in the SGA group $(p<$ 0.01). Furthermore, differences, though not statistically significant, were seen in maternal body mass index (BMI, $p=0.06)$ and infant weight $(p=0.06)$ comparing populations in the SGA groups (Table 1b). Maternal body mass index was also significantly lower in the mothers of the SGA group, within each respective population $(p<0.01)$.

\section{Cell Death ELISA}

Apoptotic activity was significantly higher in the Pakistani samples, both SGA and AGA, as compared to the Swedish placental samples $(p<0.05$, Figure 3$)$. Further analysis showed a significantly higher level of apoptosis in the Pakistani population comparing the SGA and AGA samples $(p<0.01)$, and the Swedish population comparing the SGA and AGA groups ( $p<$ 0.05).

\section{Immunohistochemical Analysis and TUNEL Assay}

There were no differences in apoptotic index for p53 comparing population sub-groups, and comparing between the SGA and AGA groups. Immunohistochemical staining for $p 53$, caspase 8 , and caspase 3 can be seen in Figures 1 and 2, showing the control group (AGA), the growth restricted group (SGA), and the positive controls, for each respective marker. Caspase 8 and caspase 3 showed significantly higher apoptotic indices in the Pakistani population (SGA and AGA groups), as compared to the Swedish population. This can be more clearly seen in Figures $\mathbf{4 a}$ and $\mathbf{4 b}$, respectively $(p<0.01)$. The figures indicate higher levels of apoptosis in the Pakistani population, as well as higher levels in the SGA groups, as compared to the AGA groups, though these differences were not statistically significant. Levels of DNA fragmentation (TUNEL) were higher in the SGA groups as compared to the AGA groups, though these differences were not statistically significant (Swedish population, $p=0.16$; Pakistani population, $p=0.33$ ). Furthermore, whilst comparing between populations, levels of DNA fragmentation were higher in the Pakistani groups as compared to the Swedish groups (SGA, $p=0.06$; AGA, $p<0.01$; Figure 4c).

\section{DISCUSSION}

The aims of this study were to quantify levels of placental apoptosis comparing growth outcomes in 


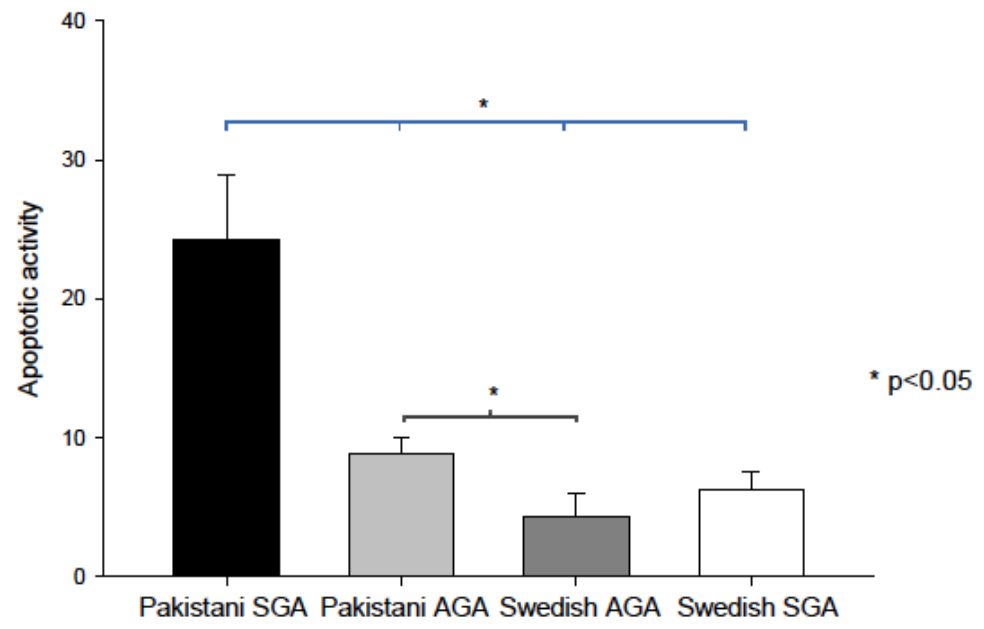

Figure 3: Comparison of apoptotic activity (enrichment factor) in placenta of the populations sub-groups using cell death ELISA. The histogram shows apoptotic activity in the SGA (Pakistani $n=12$; Swedish $n=7$ ) and AGA (Pakistani $n=12 ;$ Swedish $n=5)$ groups, comparing between populations $\left({ }^{*} p<0.05\right)$. All charts represent means with their standard errors (SEM).

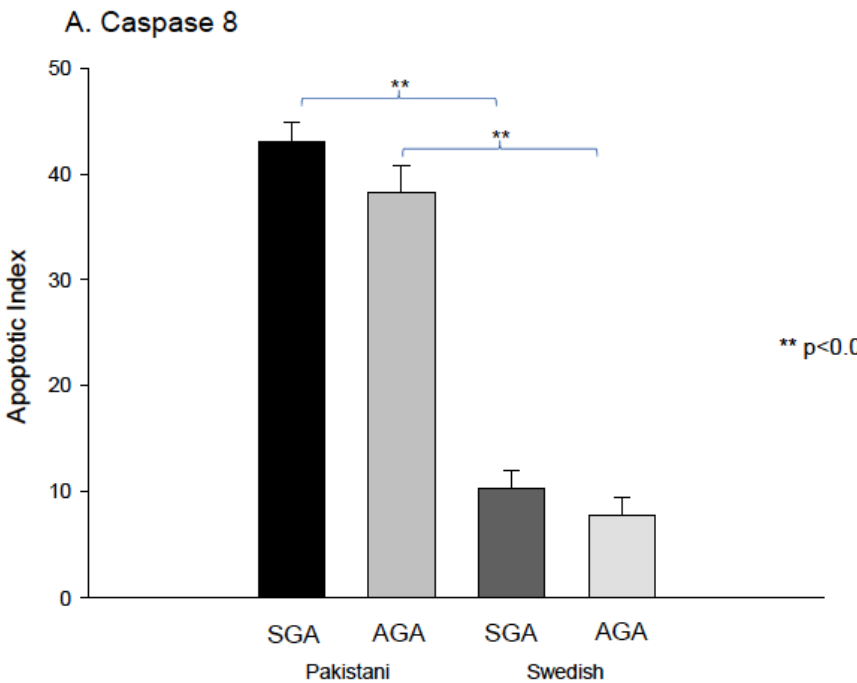

C. TUNEL
B. Caspase 3

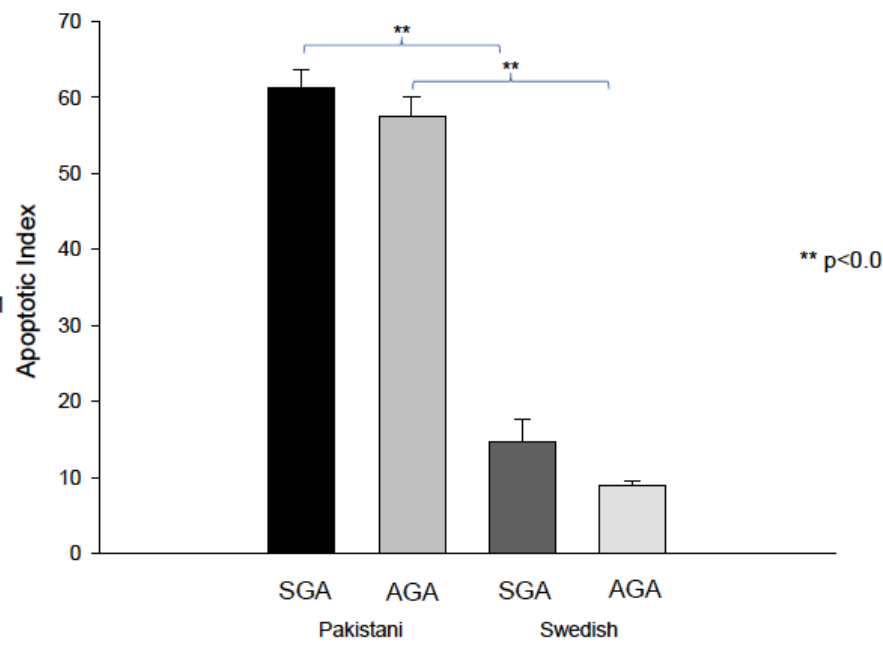

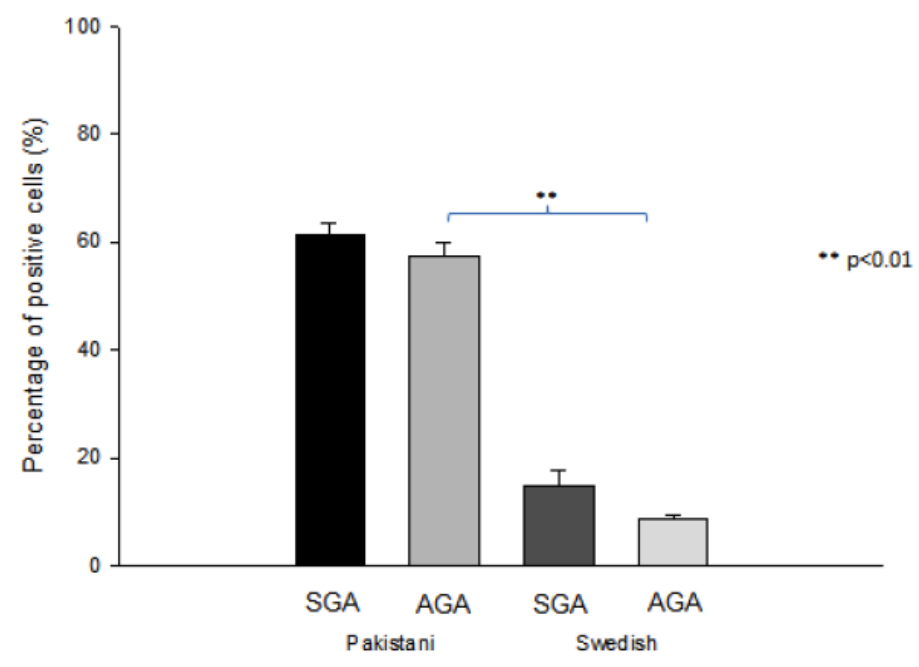

Figure 4: Histograms representing the apoptotic index for caspase 8 and caspase 3 (relative degree of apoptosis), and relative proportion of DNA fragmentation (TUNEL), in the SGA and AGA groups (Pakistani and Swedish populations). Figure 3a. Caspase 8. Figure $\mathbf{3 b}$. Caspase 3. Figure 3c. TUNEL. Significant differences were seen between the two populations $\left({ }^{* *} p<\right.$ 0.01). All indices are represented with their standard errors (SEM). 
Pakistani and Swedish population sub-groups in an attempt to better understand the mechanisms of growth restriction. Furthermore, we attempted to ascertain which apoptotic pathway was triggered in the placenta by looking at key initiators of apoptosis, namely, p53, and caspase 8 . Our results show increases in apoptotic activity in the placenta of babies born SGA, as well as higher levels of apoptotic activity in the placenta from the Pakistani population, as compared to the Swedish population. Furthermore, we have shown that the extrinsic pathway of apoptosis, through caspase 8 activation, appears to be the principal pathway of apoptosis in the placenta.

The difference in apoptotic activity between populations is interesting and may suggest a relationship between maternal size and levels of placental apoptotic activity in an attempt to control foetal-placental growth [13]. The smaller Pakistani mothers, on average, give birth to smaller babies, and this may, hypothetically, be a result of increased placental apoptosis. These results suggest the role of population genetics and environmental factors in birth weight outcomes, particularly that of maternal constraint. Gluckman, et al., describe maternal constraint as 'a set of poorly defined processes by which maternal and uteroplacental factors act to limit the growth of the foetus, presumably by limiting nutrient availability and/or the metabolic-hormonal drive to grow' [14]. They further associate this clinical phenomenon to smaller maternal size, as was seen in the SGA groups of both populations. Smaller mothers amount to smaller pelvis size and structure and thus cannot accommodate larger babies, possibly controlling foetal-placental growth and size. Smaller parents are also genetically more likely to have smaller children, though our results showed no differences in anthropometric parameters of babies born in the AGA groups comparing both populations, suggesting the importance of other factors beyond population genetics influencing foetal-placental growth [15]. We have also shown that placental apoptosis is principally controlled through the extrinsic pathway, through the activation of caspase 8 . The significantly increased apoptotic index in the Pakistani population may indicate that these placentas have a higher baseline level of apoptosis, particularly towards the end of pregnancy. They may also indicate differences in the handling of tissues from both populations, whereby small delays in the appropriate transporting and storing of tissues influences levels of apoptosis and tissue necrosis.
Barrio, et al., amongst others, has shown significantly higher levels of caspase 3 , the apoptotic executioner, in placenta of SGA babies [16, 17]. We did not, however, find any significant differences in our population groups. Furthermore, despite the lack of difference in caspase 3 positive cells in our population subgroups, we have shown significant differences in levels of DNA histone complexes in our subgroups, through the use of the apoptosis ELISA, as well as differences in DNA fragmentation through TUNEL assays. These results indicate the final products of apoptosis and suggest higher levels of apoptosis in the SGA groups as compared to the AGA groups, and in the Pakistani subgroup in comparison to the Swedish subgroup. There is little data on the direct comparison of placental apoptosis in different populations, suggesting that the significant differences we have shown in our sub-groups may in part be due to ethnic factors. As mentioned previously, comparisons of the normal control groups (AGA) between populations showed no difference, indicating that genetics may not play a significant role in the pathogenesis of FGR. We appreciate the limitations our small sample size may pose and suggest further studies be conducted to compare apoptosis across populations.

Protein 53 (p53) is a well known pro-apoptotic tumour suppressor and has been shown to promote growth arrest and cellular senescence [18]. Furthermore, it has also been shown to block angiogenesis, restricting tissue growth. Increases in p53 gene expression have been further associated with the development of gestational trophoblastic disease and trophoblastic apoptosis in association with foetal growth restriction $[19,20]$. P53 may thus act as an intrinsic initiator of apoptosis in higher risk pregnancies. We did not, however, find any p53 positive cells in our subgroups, suggesting that the placental activation of apoptosis is principally regulated through the extrinsic pathway, possibly through decreased expression of growth promoting factors, as has previously been described by us [11, 21]

The IGF-axis plays a key role in foetal-placental growth, with down-regulation resulting in increases in placental apoptosis as a possible cause for intrauterine growth restriction [22]. Furthermore, IGF-I has been previously shown to have anti-apoptotic activity through the activation of an intracellular tyrosine kinase receptor, playing a key role in cell and tissue growth and modulation [23]. We have previously shown similar results of the effects of decreased IGF-I and IGF-II 
mRNA expression, along with decreased IGF-I protein levels, correlating to low birth weight [21]. The increased apoptotic activity in the placenta of SGA babies, seen in our results, suggests that this increase may be secondary to the down-regulation of the IGFaxis, thereby resulting in decreased growth and subsequent growth restriction. Furthermore, this fine balance between growth promoting and suppressing factors appears to be associated with maternal anthropometry, re-emphasising the importance of establishing maternal pregnancy growth charts to aid in the timely diagnosis of intrauterine growth restriction (IUGR). This is of particular importance in areas where ethnic and environmental factors appear to play a role in the pathogenesis of this devastating clinical problem. In developing countries, such as Pakistan, where advanced radiological diagnostic tools are scarcely available, early screening and identification of high-risk pregnancies through clinical screening may allow for timely intervention. This, in turn, may help break this vicious cycle of poor growth, aiding towards a healthier population. Finally, maternal blood samples screening for biomarkers of foetal-placental growth (such as placental growth factors) may be an additional useful screening tool, globally, to help improve the diagnosis of IUGR and pregnancy related disorders affecting foetal-placental growth, such as pre-eclampsia [24].

\section{ACKNOWLEDGEMENTS}

We thank Drs. Farasat Zaman and Eugenia Colon for their technical expertise. This work was financially supported by the Foundation for Paediatric Endocrinology Research, Stiftelsen Frimurare Barnhuset in Stockholm, Sällskapet Barnavård, the Swedish Research Council (projects \# 8282 and 20137), and the Karolinska Institutet.

\section{REFERENCES}

[1] de Onis M, Blossner M, Villar J. Levels and patterns of intrauterine growth retardation in developing countries. Eur $\mathrm{J}$ Clin Nutr 1998; 52(Suppl 1): S5-15.

[2] Haram K, Gjelland K. [Foetal growth retardation]. Tidsskr Nor Laegeforen 2007; 127(20): 2665-9.

[3] Fowden AL. The insulin-like growth factors and feto-placental growth. Placenta 2003; 24(8-9): 803-12. http://dx.doi.org/10.1016/S0143-4004(03)00080-8

[4] Chatelain P. Children born with intra-uterine growth retardation (IUGR) or small for gestational age (SGA): long term growth and metabolic consequences. Endocr Regul 2000; 34(1): 33-6.

[5] Austgulen R, Isaksen CV, Chedwick L, Romundstad P, Vatten L, Craven C. Pre-eclampsia: associated with increased syncytial apoptosis when the infant is small-forgestational-age. J Reprod Immunol 2004; 61(1): 39-50. http://dx.doi.org/10.1016/j.jir.2003.10.001
Randhawa $\mathrm{R}$, Cohen $\mathrm{P}$. The role of the insulin-like growth factor system in prenatal growth. Mol Genet Metab 2005; 86(1-2): 84-90

http://dx.doi.org/10.1016/j.ymgme.2005.07.028

[7] Jin Z, El-Deiry WS. Overview of cell death signaling pathways. Cancer Biol Ther 2005; 4(2): 139-63.

http://dx.doi.org/10.4161/cbt.4.2.1508

[8] Polyak K, Xia Y, Zweier JL, Kinzler KW, Vogelstein B. A model for p53-induced apoptosis. Nature 1997; 389(6648): 300-5. http://dx.doi.org/10.1038/38525

[9] Ashkenazi A, Dixit VM. Death receptors: signaling and modulation. Science 1998; 281(5381): 1305-8. http://dx.doi.org/10.1126/science.281.5381.1305

[10] Porter AG, Janicke RU. Emerging roles of caspase-3 in apoptosis. Cell Death Differ 1999; 6(2): 99-104. http://dx.doi.org/10.1038/sj.cdd.4400476

[11] Akram SK, Sahlin L, Ostlund E, Hagenas L, Fried G, Soder O. Placental IGF-I, estrogen receptor, and progesterone receptor expression, and maternal anthropometry in growthrestricted pregnancies in the Swedish population. Horm Res Paediatr 2011; 75(2): 131-7. http://dx.doi.org/10.1159/000320466

[12] Akram SK, Carlsson-Skwirut C, Bhutta ZA, Soder $O$ Placental IGF-I, IGFBP-1, zinc, and iron, and maternal and infant anthropometry at birth. Acta Paediatr 2011; 100(11): 1504-9. http://dx.doi.org/10.1111/j.1651-2227.2011.02336.x

[13] Proos LA. Anthropometry in adolescence--secular trends, adoption, ethnic and environmental differences. Horm Res 1993; 39(Suppl 3): 18-24. http://dx.doi.org/10.1159/000182781

[14] Gluckman PD, Hanson MA. Maternal constraint of fetal growth and its consequences. Semin Fetal Neonatal Med 2004; 9(5): 419-25. http://dx.doi.org/10.1016/..siny.2004.03.001

[15] Visscher PM. Sizing up human height variation. Nat Genet 2008; 40(5): 489-90.

http://dx.doi.org/10.1038/ng0508-489

[16] Agata KB, Anita S, Urszula KK, Agnieszka NK, Grzegorz B. Expression of caspase-3, Bax nad Bcl-2 in placentas from pregnancies complicated by treated and non-treated fetal growth restriction. Ginekol Pol 2009; 80(9): 652-6.

[17] Barrio E, Calvo MT, Miramar MD, Lorente F, Rodriguez A Labarta Jl, et al. Study of apoptosis and related proteins, $\mathrm{CRH}$ and $\mathrm{hpGH}$ in placentas of newborns small for gestational age (SGA). Pediatr Endocrinol Rev 2009; 6(Suppl 3): 337-42.

[18] Amaral JD, Xavier JM, Steer CJ, Rodrigues CM. Targeting the p53 pathway of apoptosis. Curr Pharm Des 2010; 16(22): 2493-503.

http://dx.doi.org/10.2174/138161210791959818

[19] Halperin R, Peller S, Sandbank J, Bukovsky I, Schneider D. Expression of the p53 gene and apoptosis in gestational trophoblastic disease. Placenta 2000; 21(1): 58-62. http://dx.doi.org/10.1053/plac.1999.0442

[20] Levy R, Smith SD, Yusuf K, Huettner PC, Kraus FT, Sadovsky $\mathrm{Y}$, et al. Trophoblast apoptosis from pregnancies complicated by fetal growth restriction is associated with enhanced p53 expression. Am J Obstet Gynecol 2002; 186(5): 1056-61. http://dx.doi.org/10.1067/mob.2002.122250

[21] Akram SK, Akram M, Bhutta ZA, Soder O. Human placental IGF-I and IGF-II expression: correlating maternal and infant anthropometric variables and micronutrients at birth in the Pakistani population. Acta Paediatr 2008; 97(10): 1443-8. http://dx.doi.org/10.1111/j.1651-2227.2008.00930.x

[22] Ain R, Canham LN, Soares MJ. Dexamethasone-induced intrauterine growth restriction impacts the placental prolactin 
family, insulin-like growth factor-II and the Akt signaling pathway. J Endocrinol 2005; 185(2): 253-63. http://dx.doi.org/10.1677/joe.1.06039

[23] Laviola L, Natalicchio A, Giorgino F. The IGF-I signaling pathway. Curr Pharm Des 2007; 13(7): 663-9. http://dx.doi.org/10.2174/138161207780249146
[24] Litton C, Stone J, Eddleman K, Lee MJ. Noninvasive prenatal diagnosis: past, present, and future. Mt Sinai J Med 2009; 76(6): 521-8.

http://dx.doi.org/10.1002/msj.20153

Received on 06-03-2014

Accepted on 12-04-2014

Published on 30-05-2014

http://dx.doi.org/10.6000/1929-4247.2014.03.02.5

(C) 2014 Akram et al.; Licensee Lifescience Global.

This is an open access article licensed under the terms of the Creative Commons Attribution Non-Commercial License (http://creativecommons.org/licenses/by-nc/3.0/) which permits unrestricted, non-commercial use, distribution and reproduction in any medium, provided the work is properly cited. 